NRCPS-HE-38-2011

CERN-PH-TH/2011-175

\title{
Conformal Invariance of Tensor Boson Tree Amplitudes
}

\author{
Ignatios Antoniadis ${ }^{1 *}$ and George Savvidy ${ }^{1,3}$ \\ ${ }^{1}$ Department of Physics, CERN Theory Division CH-1211 Geneva 23, Switzerland \\ ${ }^{3}$ Demokritos National Research Center, Ag. Paraskevi, Athens, Greece
}

\begin{abstract}
The BCFW recursion relation allows to find out the tree-level scattering amplitudes for gluons and tensor gauge bosons in generalized Yang-Mills theory. We demonstrate that the corresponding MHV amplitudes for the tensor gauge bosons of spin- $s$ and $n$ gluons are invariant under conformal group of transformations. This is highly unexpected result for the higher spin particles, in particular this is not true for the scattering amplitudes of gravitons. We discuss and compare the tree-level scattering amplitudes for the charged tensor bosons with the corresponding scattering amplitudes for gravitons, stressing their differences and similarities.
\end{abstract}

\footnotetext{
* On leave of absence from CPHT École Polytechnique, F-91128, Palaiseau Cedex,France.
} 


\section{$1 \quad$ Introduction}

The Lagrangian of non-Abelian tensor gauge fields describes the interaction of the gluons with their massless excitations of higher spin [1, 2, 3]. The characteristic property of generalized Yang-Mills theory is that all interaction vertices between gluons and their high-spin excitations have dimensionless coupling constants in four-dimensional space-time. That is, the cubic interaction vertices have only first order derivatives and the quartic vertices have no derivatives at all.

For better understanding of the model it is important to study the corresponding tree level scattering amplitudes. One of the first calculations of tree level amplitudes was made in the article [26] where the authors considered the production of tensor gluons in the annihilation processes of quarks and gluons. It uses a very powerful spinor helicity technique and color decomposition for the calculation of high order tree level diagrams in Yang-Mills and other supersymmetric theories [4, 5, 6, 7, 8, 9, 10, 11, 17, 18, 19, 20, 21, 22, 23, 24, 25]. The spinor and twistor [11, 12, 13, 14, 15, 16] representations of the scattering amplitudes dramatically simplifies the calculations [8, 17, 18, 19]. The application of the BCFW recurrence relations [17, 18] to calculate four-particle amplitudes allows to derive the production rate of tensor gluons of arbitrary high spin in the fusion of two gluons $g+g \rightarrow T+T$ [26]. The consistency of the calculations in different kinematical channels is fulfilled when all cubic coupling constants between vector bosons (gluons) and high spin tensor bosons are of the generalized Yang-Mills type [1, 2] and are equal to the Yang-Mills gauge coupling $g=g_{Y M}$. The result can be expressed in a compact form [26]:

$$
d \sigma_{g_{+} g_{-} \rightarrow T_{+} T_{-}}=\left(\frac{1-\cos \theta}{1+\cos \theta}\right)^{2 s-2} d \sigma_{g_{+} g_{-} \rightarrow g_{+} g_{-}}, \quad s=1,2,3, \ldots
$$

where $d \sigma_{g_{+} g_{-} \rightarrow g_{+} g_{-}}$is the polarized cross section of two gluons into two gluons and $\theta$ is the scattering angle. The formula demonstrates the complete dependence of the cross section on the spin of the tensor gluons through the form-factor $F(\theta)^{2 s-2}$

$$
F(\theta)=\frac{1-\cos \theta}{1+\cos \theta}
$$

in front of the gluon fusion cross section $d \sigma_{g_{+} g_{-} \rightarrow g_{+} g_{-}}$.

One can also derive a generalization of the Parke-Taylor scattering amplitude to the case of two tensor gauge bosons of spin $s$ and $(n-2)$ gluons. The result of Georgiou-Savvidy 
reads [26]:

$$
M_{n}\left(1^{+}, . . i^{-}, . . k^{+s}, . . j^{-s}, . . n^{+}\right)=g^{n-2} \frac{<i j>^{4}}{\prod_{r=1}^{n}<r(r+1)>}\left(\frac{<i j>}{<i k>}\right)^{2 s-2},
$$

where $n$ is the total number of particles, and the dots stand for the positive helicity gluons. Here, $i$ is the position of the negative helicity gluon, while $k$ and $j$ are the positions of the particles with helicities $+s$ and $-s$ respectively. This expression is holomorphic in the spinor dependence, exactly as the MHV gluon amplitude and for $s=1$ the second fraction in (1.3) is absent and (1.3) reduces to the well-known result for the MHV amplitude [9]. It is also remarkable that the formula correctly reproduces the tree level gluon scattering amplitudes with the insertion of quark or scalar pair instead of tensor particles. Indeed this take place when we substitute $s=1 / 2$ or $s=0$ in (1.3). In a sense the formula has larger validity area than the area in which it has been initially derived [26].

The next generalization of scattering amplitudes for high helicity particles can be obtained by considering two pairs of tensor gauge bosons of spin $s_{1}$ and $s_{2}$ and $(n-4)$ gluons. The corresponding amplitude which we have found has the form

$$
M_{n}\left(1^{+}, . . l^{+s_{1}}, . . i^{-s_{1}}, . . k^{+s_{2}}, . . j^{-s_{2}}, . . n^{+}\right)=g^{n-2} \frac{<i j>^{4}}{\prod_{r=1}^{n}<r r+1>}\left(\frac{<i j>}{<i k>}\right)^{2 s_{2}-2}\left(\frac{<i j>}{<l j>}\right)^{2 s_{1}-2}
$$

and is holomorphic in the spinors of the particles, it reduces to the amplitude (1.3) when $s_{1}=1$ or when $s_{2}=1$ and to the Parke-Taylor amplitude when both spins are equal to one. The derivation of the amplitude (1.4) will be presented elsewhere, here we shall limit ourself in studying its conformal properties.

It is well known that the MHV Parke-Taylor tree amplitudes [9] are conformal invariant [11] and that at the loop-level the conformal symmetry is broken in non-supersymmetric gauge theories like QCD. In $\mathcal{N}=4$ SYM the situation is different, it is fully superconformal theory where the redefinition of the superconformal generators is required in order to restor the conformal symmetry at the loop-level [16]. Our intension is to study the conformal properties of the generalized tree-level amplitudes (1.3) and (1.4) which are describing the scattering of the tensor gauge bosons. We shall demonstrate that these generalized tree amplitudes (1.3) and (1.4) are conformal invariant + . This is highly unexpected result, as

\footnotetext{
${ }^{\dagger}$ A tree-level holomorphic anomaly [12, 13, 14, 15, 16, caused by the collinear momenta, can be avoided for tree amplitudes through a choice of external momenta in a general position.

${ }_{\ddagger}^{\ddagger}$ The interplay between scale and conformal symmetries in quantum field theory recently were discussed in a number of publications [29, 30, 31, 32, 33, 34, 35.
} 
we shell discuses in the main text of the article that this is not true in particular for the scattering amplitudes of gravitons. The problem of conformal invariance of higher spin gauge theories is an old and unsolved problem even at the classical level and the above result can shed some light on that difficult problem. The higher spin extension of the conformal group have been found recently in [3], but it is not yet known if we have here an example of its field theoretical realization. It is difficult to expect that the scattering amplitudes of the tensor gauge bosons will remain conformal invariant at the loop-level and most probably we shall have the breaking of conformal symmetry similar to the QCD case.

We shall also discuss and compare the above amplitudes for the charged tensor gauge bosons with the corresponding amplitudes for gravitons, stressing their differences and similarities. The differences have their origin in the charge content of the particles and in the form of the basic cubic interaction vertices in the corresponding theories, while the similarity lies in the helicity content of the particles.

\section{$2 \quad$ Helicity Amplitudes and Structure of Interaction Vertices}

Let us consider a scattering amplitude for massless particles of momenta $p_{i}$ and polarization tensors $\varepsilon_{i}(i=1, \ldots, n)$, which are described by irreducible massless representations of Poincaré group and are classified by their helicities $h= \pm s$, where $s$ is an integer:

$$
M_{n}=M_{n}\left(p_{1}, \varepsilon_{1} ; p_{2}, \varepsilon_{2} ; \ldots ; p_{n}, \varepsilon_{n}\right) .
$$

We are interested in representing the momenta $p_{i}$ and polarization tensors $\varepsilon_{i}$ in terms of spinors and the above scattering amplitude in terms of rational functions of spinor products [4, 5, 6, 17, 8, 9, 10, 11, 17, 18, 19, 20, 21, 22, 23, 24, 25].

The spinor representation of momenta $p^{\mu}$ and polarization tensors $\varepsilon_{i}$ can be constructed as follows. The spinors $\left\{\lambda_{a}, \tilde{\lambda}_{\dot{a}}\right\}$ transform in the representation $(1 / 2,0)$ and $(0,1 / 2)$ of the universal cover of the Lorentz group, $S L(2, C)$, respectively. Invariant tensors are $\epsilon^{a b}$, $\epsilon^{\dot{a} \dot{b}}$ and $\left(\sigma^{\mu}\right)_{a \dot{a}}$, where $\sigma^{\mu}=(1, \vec{\sigma})$. The basic Lorentz invariant spinor products can be constructed as follows: $\lambda_{a} \lambda_{b}^{\prime} \epsilon^{a b} \equiv<\lambda, \lambda^{\prime}>, \tilde{\lambda}_{\dot{a}} \tilde{\lambda}_{\dot{b}}^{\prime} \epsilon^{\dot{a} \dot{b}} \equiv\left[\lambda, \lambda^{\prime}\right]$. The scalar product of two vectors $p^{\mu}$ and $q^{\nu}$ is given by the product $2(p \cdot q)=<\lambda^{p}, \lambda^{q}>\left[\tilde{\lambda}^{p}, \tilde{\lambda}^{q}\right]$. Using the third invariant tensor one can define $p^{\mu}=\lambda^{a}\left(\sigma^{\mu}\right)_{a \dot{a}} \tilde{\lambda}^{\dot{a}}$ and find out the corresponding spinor 
representation of massless particle momentum in the form

$$
p_{a \dot{a}}=\lambda_{a} \tilde{\lambda}_{\dot{a}}
$$

The corresponding polarization vectors of spin-1 particles are given by

$$
\varepsilon_{a \dot{a}}^{-}(p)=\frac{\lambda_{a} \tilde{\mu}_{\dot{a}}}{[\tilde{\lambda}, \tilde{\mu}]}, \quad \varepsilon_{a \dot{a}}^{+}(p)=\frac{\mu_{a} \tilde{\lambda}_{\dot{a}}}{<\mu, \lambda>}
$$

with $\mu_{a}$ and $\tilde{\mu}_{\dot{a}}$ as arbitrary reference spinors. The polarization tensors of massless particles of integer spin $s$ can be expressed in terms of spin-1 as follow $\$$ :

$$
\varepsilon_{a_{1} \dot{a}_{1}, \ldots, a_{s} \dot{a}_{s}}^{-}(p)=\prod_{i=1}^{s} \varepsilon_{a_{i} \dot{a}_{i}}^{-}, \quad \varepsilon_{a_{1} \dot{a}_{1}, \ldots, a_{s} \dot{a}_{s}}^{+}(p)=\prod_{i=1}^{s} \varepsilon_{a_{i} \dot{a}_{i}}^{+}
$$

The presence in (2.7) of arbitrary reference spinors $\mu_{a}$ and $\tilde{\mu}_{\dot{a}}$ means that polarization tensors are not uniquely fixed and are changing under the gauge transformations.

The scattering amplitude of massless bosons $M_{n}(2.5)$ can be considered now as a function of spinors $\lambda_{i}, \tilde{\lambda}_{i}$ and helicities $h_{i}: M_{n}=M_{n}\left(\lambda_{1}, \tilde{\lambda}_{1}, h_{1} ; \ldots ; \lambda_{n}, \tilde{\lambda}_{n}, h_{n}\right)$ and should be a homogeneous function of these spinors of order $2 h_{i}$ [11]:

$$
\left(\lambda_{i}^{a} \frac{\partial}{\lambda_{i}^{a}}-\tilde{\lambda}_{i}^{a} \frac{\partial}{\tilde{\lambda}_{i}^{a}}\right) M_{n}\left(\ldots\left\{\lambda_{i}, \tilde{\lambda}_{i}, h_{i}\right\} \ldots\right)=-2 h_{i} M_{n}\left(\ldots\left\{\lambda_{i}, \tilde{\lambda}_{i}, h_{i}\right\} \ldots\right) .
$$

From this equation one can derive a general structure of the full three-particle amplitudes $M_{3}$ in spinor representation for the complex momenta [11, 19]. This provides a unique information about the structure of three-point on-shell vertices $M_{3}\left(p_{1}, p_{2}, p_{3}\right)$ in high spin quantum field theory. Indeed, looking for a polynomial solution one can find that the three-point on-shell vertices for complex momenta have the form 9 [19, 26]

$$
\begin{aligned}
M_{3}\left(1^{h_{1}}, 2^{h_{2}}, 3^{h_{3}}\right)= & f<1,2>^{h_{3}-h_{1}-h_{2}}<2,3>^{h_{1}-h_{2}-h_{3}}<3,1>^{h_{2}-h_{3}-h_{1}}+ \\
& k[1,2]^{-h_{3}+h_{1}+h_{2}}[2,3]^{-h_{1}+h_{2}+h_{3}}[3,1]^{-h_{2}+h_{3}+h_{1}},
\end{aligned}
$$

where $f$ and $k$ are momentum independent constants and they are such that if $h_{1}+h_{2}+h_{3}<$ 0 , then $k=0$, while if $h_{1}+h_{2}+h_{3}>0$ then $f=0$ in order to avoid singularities in the limit

\footnotetext{
$\S$ In labeling helicities, we consider all particles to be outgoing.

IThe earlier investigation of the three-point vertices in the light-front formulation of relativistic dynamics and derivation of restrictions imposed on the helicities of scattered particles by the Poincaré group were made in [36, 37, 38, 39. In covariant formulation the interaction vertices were studied in 40, 41, 42, see also [43, 44, 45, 46, 47, 48, 49, 50, 51, 52, 53. The advantage of the spinor formulation is that it gives non-perturbative expressions.
} 
of the real momenta. Because the dimensionality of spinors $\lambda$ and $\tilde{\lambda}$ in formula (2.6) is $[\text { mass }]^{1 / 2}$, the dimensionality of the three-point on-shell vertex $M_{3}$ is $[\text { mass }]^{D=\mp\left(h_{1}+h_{2}+h_{3}\right)}$.

In the generalized Yang-Mills theory [1, 2] all interaction vertices between high-spin fields have dimensionless coupling constants in four-dimensional space-time, therefore one should impose the constraint $D=\mp\left(h_{1}+h_{2}+h_{3}\right)=1$ on the amplitudes $M_{3}$ in (2.10), which gives [26]:

$$
\begin{array}{ll}
M_{3}=f<1,2>^{-2 h_{1}-2 h_{2}-1}<2,3>^{2 h_{1}+1}<3,1>^{2 h_{2}+1}, & h_{3}=-1-h_{1}-h_{2}, \\
M_{3}=k[1,2]^{2 h_{1}+2 h_{2}-1}[2,3]^{-2 h_{1}+1}[3,1]^{-2 h_{2}+1}, & h_{3}=1-h_{1}-h_{2} .
\end{array}
$$

The formulas (2.11) give a general expression for the dimensionless vertex $M_{3}$ in terms of two independent helicities $h_{1}$ and $h_{2}$ in the generalized Yang-Mills theory [1, 2]. In particular, one can derive nontrivial subclass of interaction vertices which contains at least one vector boson of spin 1 and higher-spin bosons which have dimensionless coupling constant [1, 56, 57, 2, 26]. Taking in (2.11) $h_{1}= \pm 1$ and $h_{2}= \pm s$ one can find $h_{3}$ from the equations

$$
h_{1}+h_{2}+h_{3}= \pm 1
$$

The six corresponding solutions are

$$
h_{3}= \pm|s-2|, \pm s, \pm|s+2|
$$

and the typical vertex has the form

$$
M_{3}^{a_{1} a_{2} a_{3}}\left(1^{-s}, 2^{-1}, 3^{+s}\right)=g f^{a_{1} a_{2} a_{3}} \frac{<1,2>^{4}}{<1,2><2,3><3,1>}\left(\frac{<1,2>}{<2,3>}\right)^{2 s-2},
$$

and contains a vector and two spin-s bosons. We have also a vertex which contains spin- $s$ and spin- $|s-2|$ bosons:

$$
M_{3}^{a_{1} a_{2} a_{3}}\left(1^{-s}, 2^{s-2}, 3^{+1}\right)=g f^{a_{1} a_{2} a_{3}} \frac{<1,2>^{4}}{<1,2><2,3><3,1>}\left(\frac{<1,3>}{<2,3>}\right)^{2 s-2},
$$

where $f^{a b c}$ are the structure constants of the internal gauge group G. These vertices reduce to the standard Yang-Mills vertex when $s=1$ and can be written in a factorized form

$$
\begin{gathered}
M_{3}^{a_{1} a_{2} a_{3}}\left(1^{-s}, 2^{-1}, 3^{+s}\right)=M_{3}^{Y M a_{1} a_{2} a_{3}}\left(1^{-1}, 2^{-1}, 3^{+1}\right)\left(\frac{<1,2>}{<2,3>}\right)^{2 s-2}, \\
M_{3}^{a_{1} a_{2} a_{3}}\left(1^{-s}, 2^{s-2}, 3^{+1}\right)=M_{3}^{Y M a_{1} a_{2} a_{3}}\left(1^{-1}, 2^{-1}, 3^{+1}\right)\left(\frac{<1,3>}{<2,3>}\right)^{2 s-2} .
\end{gathered}
$$


The vertex which does not reduce to the Yang-Mills one contains spin-s and spin- $|s+2|$ bosons and has the formll

$$
M_{3}^{a_{1} a_{2} a_{3}}\left(1^{-(s+2)}, 2^{s}, 3^{+1}\right)=g f^{a_{1} a_{2} a_{3}} \frac{<1,2>^{4}}{<1,2><2,3><3,1>}\left(\frac{<1,3>}{<2,3>}\right)^{2 s+2} .
$$

These are the primitive vertices in the sense that all others can be obtained by the symmetries such as parity and cyclic $Z_{3}$ symmetry, which allow simultaneously to reverse all helicities in a vertex and cyclically exchange their positions. In summary, the above vertices can be written in a factorized form with a nontrivial prefactor depending on helicities involved, and in the general case we can represent the vertex (2.11) in the same factorized form $\left(h_{1}+h_{2}+h_{3}=-1\right)$

$$
M_{3}^{a_{1} a_{2} a_{3}}\left(1^{h_{1}}, 2^{h_{2}}, 3^{h_{3}}\right)=M_{3}^{Y M a_{1} a_{2} a_{3}}\left(1^{-1}, 2^{-1}, 3^{+1}\right)\left(\frac{<2,3>}{<1,2>}\right)^{2 h_{1}+2}\left(\frac{<3,1>}{<1,2>}\right)^{2 h_{2}+2}
$$

explicitly exhibiting its dimensionless character.

It is now tempting to present the interaction vertex in gravity where all helicities are equal to $h_{i}= \pm 2$ and compare it with the Yang-Mills one [54, 55, 23, 24]. Using the general formula (2.10) one can get the cubic vertex for the neutral gravitons:

$$
M_{3}^{G R}\left(1^{-2}, 2^{-2}, 3^{+2}\right)=\kappa \frac{<1,2>^{8}}{<1,2>^{2}<2,3>^{2}<3,1>^{2}}=\kappa\left(M_{3}^{Y M}\left(1^{-1}, 2^{-1}, 3^{+1}\right)\right)^{2}
$$

which has dimensionful coupling constant $\kappa^{2}=32 \pi G_{N}$ in front of the quadratic in momenta spinor expression; one sees that it is exactly equal to the square of the color/charge stripped gluon vertex $M_{3}^{Y M}$. The other vertex we are interested in is the graviton-photon vertex of helicities $(-2,-1,+1)$

$$
: M_{3}^{M G R}\left(1^{-2}, 2^{-1}, 3^{+1}\right)=\kappa \frac{<1,2>^{4}}{<2,3>^{2}}
$$

which is also quadratic in momentum and has dimensionful coupling constant.

It is now elucidating to compare interaction vertices which include neutral gravitons $\mathrm{G}$ on the one hand and charged non-Abelian tensor bosons $\mathrm{T}$ on the other hand. Both have the same helicities $h= \pm 2$, but the former are neutral while the others are charged. This results in completely different properties of the interaction vertices. The graviton vertices include odd number of gravitons (GGG) (2.17) and even number of vector bosons

\footnotetext{
${ }^{\|}$Formally it reduces to the Yang-Mills vertex if one substitutes $s=-1$, sort of analytical continuation of the angular momentum.
} 
(GVV) (2.18) and the coupling constant is dimensionful, while the charged tensor bosons vertices include even number of tensors and odd number of vector bosons (TTV) (2.16) with dimensionless coupling constant. The differences will become more transparent when we compare the tree level amplitudes in the corresponding theories in the next sections.

\section{$3 \quad$ High Spin Helicity Amplitudes}

With these vertices in hand one can compute the gluon fusion amplitudes into two high spin-s gauge bosons $s=2,3, \ldots$ For the four-particle scattering amplitudes one can find the following expression [26]:

$$
\begin{aligned}
M_{4}^{a b c d}\left(1^{+}, 2^{-}, 3^{+s}, 4^{-s}\right) & =-2 i g^{2}\left(\frac{<2,4>}{<2,3>}\right)^{2 s-2} \times \\
& \left(f^{a d e} f^{b c e} \frac{<2,4>^{4}}{\prod<i, i+1>}+f^{a c e} f^{b d e} \frac{<2,4>^{4}}{\prod_{3 \leftrightarrow 4}<i, i+1>}\right),
\end{aligned}
$$

where in the brackets of the second line above is the gluonic amplitude $g_{+}+g_{-} \rightarrow g_{+}+g_{-}$ times the form-factor which is the contribution of the high spin gauge bosons. This is purely holomorphic expression. The alternative helicity amplitude $M_{4}^{a b c d}\left(1^{+}, 1^{-}, 3^{-s}, 4^{+s}\right)$ can also be found [26]. Here the four gluon scattering amplitudes without color factors in (3.19) (the color ordered amplitudes) have the form

$$
\begin{aligned}
& M_{4}^{Y M}\left(1^{+1}, 2^{-1}, 3^{+1}, 4^{-1}\right)=\frac{<2,4>^{4}}{\prod<i, i+1>}, \\
& M_{4}^{Y M}\left(1^{+1}, 2^{-1}, 4^{-1}, 3^{+1}\right)=\frac{<2,4>^{4}}{\prod_{3 \leftrightarrow 4}<i, i+1>} .
\end{aligned}
$$

We shall also present the four graviton scattering amplitude [54, 55, 23, 24]

$$
M_{4}^{G R}\left(1^{+2}, 2^{-2}, 3^{+2}, 4^{-2}\right)=\frac{[34]<24>^{7}}{<12>^{2}<23><34><41><3,1>},
$$

which can be expressed in terms of four gluon scattering amplitudes (3.20)

$$
M_{4}^{G R}\left(1^{+2}, 2^{-2}, 3^{+2}, 4^{-2}\right)=-s_{12} M_{4}^{Y M}\left(1^{+1}, 2^{-1}, 3^{+1}, 4^{-1}\right) \times M_{4}^{Y M}\left(1^{+1}, 2^{-1}, 4^{-1}, 3^{+1}\right),
$$

but with an additional energy factor $s_{12}=\left(p_{1}+p_{2}\right)^{2}=\left(p_{3}+p_{4}\right)^{2}$. These relations can be easily understood on dimensional ground: in gauge theory the vertex is linear in momenta while in gravity it is quadratic, therefore one should have an energy factor in front of the gauge theory amplitudes. 
The next interesting result can be obtained if one considers the color-ordered scattering amplitudes involving two tensor particles of helicity $+s$ and $-s$ respectively, one negative helicity gluon and any number of gluons with positive helicity. In the case when $s=1$ one has the MHV amplitude for the scattering of vector bosons (gluons). The expression for this amplitude is given by the famous Parke-Taylor formula [9]

$$
\hat{M}_{n}\left(1^{+}, \ldots i^{-}, \ldots, j^{-}, \ldots, n^{+}\right)=i g^{n-2}(2 \pi)^{4} \delta^{(4)}\left(P^{a \dot{b}}\right) \frac{<i j>^{4}}{\prod_{l=1}^{n}<l l+1>},
$$

where

$$
P^{a \dot{b}}=\sum_{m=1}^{n} \lambda_{m}^{a} \tilde{\lambda}_{m}^{\dot{b}}
$$

is the total momentum.

The generalization of the above formula to the case of two spin $s$ bosons and $(n-2)$ gluons has been found in [26]:

$$
\hat{M}_{n}\left(1^{+}, . . i^{-}, \ldots k^{+s}, . . j^{-s}, . . n^{+}\right)=i g^{n-2}(2 \pi)^{4} \delta^{(4)}\left(P^{a \dot{b}}\right) \frac{<i j>^{4}}{\prod_{l=1}^{n}<l l+1>}\left(\frac{<i j>}{<i k>}\right)^{2 s-2},
$$

where $n$ is the total number of particles, and the dots stand for any number of positive helicity gluons. Finally, $i$ is the position of the negative helicity gluon, while $k$ and $j$ are the positions of the particles with helicities $+s$ and $-s$ respectively. The first comment is that the expression (3.24) is holomorphic in the spinors of the particles, exactly as the MHV gluon amplitude (3.23) is. The second comment is that for $s=1$ the second fraction in (3.24) is absent and (3.24) reduces to the MHV amplitude (3.23). In particular the five-particle amplitude takes the following form**:

$$
\hat{M}_{5}\left(1^{+}, 2^{-}, 3^{+}, 4^{+s}, 5^{-s}\right)=i g^{3}(2 \pi)^{4} \delta^{(4)}\left(P^{a \dot{b}}\right) \frac{<25>^{4}}{\prod_{i=1}^{5}<i i+1>}\left(\frac{<25>}{<24>}\right)^{2 s-2} .
$$

The third comment is that the ratio of the amplitudes for the pair of tensor gauge bosons of spin $s_{1}$ and spin $s_{2}$ has the form

$$
\hat{M}_{n}\left(1^{+}, . . i^{-}, \ldots k^{+s_{1}}, . . j^{-s_{1}}, . . n^{+}\right)=\left(\frac{<i j>}{<i k>}\right)^{2 s_{1}-2 s_{2}} \hat{M}_{n}\left(1^{+}, . . i^{-}, \ldots k^{+s_{2}}, . . j^{-s_{2}}, . . n^{+}\right)
$$

and is in formal agrement with the supersymmetric Ward identities [58, 59, 8] connecting gluon or graviton amplitudes with the insertion of a pair of partons $P$

$$
A_{n}^{S U S Y}\left(1^{+}, . . i^{-}, \ldots k^{+h_{P}}, . . j^{-h_{P}}, . . n^{+}\right)=\left(\frac{<i j>}{<i k>}\right)^{2 h_{P}-2 h_{\phi}} A_{n}^{S U S Y}\left(1^{+}, . . i^{-}, \ldots k_{\phi}^{+}, . . j_{\phi}^{-}, . . n^{+}\right) \text {, }
$$

** The 4- and 5-particle amplitudes correspond to 2-jet and 3-jet production in hadronic collisions at very high energies, and so are of phenomenological interest. 
where $P$ refers to a scalar, fermion, gluon, gravitino or graviton with respective helicity $h_{P}=0,1 / 2,1,3 / 2,2$ and $\phi$ refers to a scalar particle, or graviton.

The other generalization of the scattering amplitudes for high helicity particles can be obtained by considering a pair of particles of spin $s_{1}$ and $s_{2}$ and (n-4) gluons. The corresponding amplitude has the form

$$
\begin{aligned}
& M_{n}\left(1^{+}, . . l^{+s_{1}}, . . i^{-s_{1}}, . . k^{+s_{2}}, . . j^{-s_{2}}, . . n^{+}\right)= \\
& \quad=i g^{n-2}(2 \pi)^{4} \delta^{(4)}\left(P^{a \dot{b}}\right) \frac{<i j>^{4}}{\prod_{r=1}^{n}<r r+1>}\left(\frac{<i j>}{<i k>}\right)^{2 s_{2}-2}\left(\frac{<i j>}{<l j>}\right)^{2 s_{1}-2}
\end{aligned}
$$

and is holomorphic in the spinor dependence, reduces to the amplitude (3.24) when $s_{1}=1$ or $s_{2}=1$ and reduces to the Parke-Taylor amplitude when both spins are equal to one.

The exceptionally interesting non-MHV amplitude which involves two vector bosons and two tensor bosons of different spins $s$ and $s-2$ is [26]:

$$
\begin{aligned}
M_{4}^{a b c d}(+1,-s,+1, s-2) & =-2 i g^{2}\left(\frac{<2,3>}{<3,4>}\right)^{2 s-2} \times \\
& \left(f^{a d e} f^{b c e} \frac{<2,4>^{4}}{\prod<i, i+1>}+f^{a c e} f^{b d e} \frac{<2,4>^{4}}{\prod_{3 \leftrightarrow 4}<i, i+1>}\right),
\end{aligned}
$$

where $s=2,3, \ldots$. It is interesting to notice that if one formally substitutes $s=1$ into the above expression, then one can see that it reduces to the correct Yang-Mills amplitude $M_{4}^{a b c d}(1,-1,1,-1)$, as it already happened in the case of the corresponding three-point on-shell vertex (2.15).

This amplitude is holomorphic and is of special interest because it has only one particle of negative helicity. In comparison, the $n$-gluon tree amplitudes for $n \geq 4$ with all but one gluon of positive helicity vanish [9, 11]. Thus the $n$-particle tree amplitudes in the generalized Yang-Mills theory have more complicated structure. It is well known that the tree level $n$-gluon scattering amplitudes with all positive helicities also vanish [9, 11]. In generalized Yang-Mills theory this statement remains true and can be proved by induction [26]. Thus in the generalized Yang-Mills theory the tree level n-particle scattering amplitudes with all positive helicities vanish $M_{n}^{\text {tree }}(+, \ldots,+)=0$, but tree amplitudes with one negative helicity particle are already nonzero (3.29).

Our intension in the next section is to check the conformal properties of the amplitudes (3.24) and (3.28). 


\section{Conformal Invariance of Tensor Bosons MHV Amplitudes}

We are interested now in studying the conformal properties of the above generalized MHV amplitudes (3.24). The generators of the conformal algebra expressed in terms of the $\lambda$ and $\tilde{\lambda}$ variables are given in [1] and have the following form:

$$
\begin{gathered}
P_{a \dot{b}}=\lambda_{a} \tilde{\lambda}_{\dot{a}}, \quad J_{a b}=\frac{i}{2}\left(\lambda_{a} \frac{\partial}{\partial \lambda^{b}}+\lambda_{b} \frac{\partial}{\partial \lambda^{a}}\right), \quad \tilde{J}_{\dot{a} \dot{b}}=\frac{i}{2}\left(\tilde{\lambda}_{\dot{a}} \frac{\partial}{\partial \tilde{\lambda}^{\dot{b}}}+\tilde{\lambda}_{\dot{b}} \frac{\partial}{\partial \tilde{\lambda}^{\dot{a}}}\right) \\
D=\frac{i}{2}\left(\lambda^{a} \frac{\partial}{\partial \lambda^{a}}+\tilde{\lambda}^{\dot{a}} \frac{\partial}{\partial \tilde{\lambda}^{\dot{a}}}+2\right), \quad K_{a \dot{b}}=\frac{\partial^{2}}{\partial \lambda^{a} \partial \tilde{\lambda}^{\dot{b}}}
\end{gathered}
$$

Lorentz invariance of the formula (3.24) is manifest, the momentum conservation is fulfilled because of the delta function and we have to verify that the amplitude is annihilated by $\mathrm{D}$ and by $\mathrm{K}$ generators.

The dilatation operator D annihilates MHV amplitude (3.23) [11], therefore one should check that the additional terms arising from the factor

$$
\left(\frac{<i j>}{<i k>}\right)^{2 s-2}
$$

in the amplitude (3.24) also vanish. The derivatives acting on the spinors $\lambda_{j}$ and $\lambda_{k}$ cancel each other and the derivative over the spinor $\lambda_{i}$ vanishes as well, therefore $D \hat{M}_{n}=0$.

Similarly the special conformal generator $K_{a \dot{a}}$ annihilates the MHV amplitude (3.23) [11], and one should check that it annihilates the amplitude in the presence of the formfactor (4.31). Since $\partial M_{n} / \partial \tilde{\lambda}=0$ and $J_{a b} M_{n}=0$ the main part of the calculations in [11] remains valid and we have

$$
\begin{aligned}
K_{a \dot{a}} \hat{M}_{n} & =\frac{\partial^{2}}{\partial \lambda^{a} \partial \tilde{\lambda}^{\dot{a}}} \hat{M}_{n} \\
& =i g^{n-2}(2 \pi)^{4}\left((n-4) M_{n} \frac{\partial}{\partial P^{a \dot{a}}} \delta^{(4)}(P)+\left(\sum_{m} \lambda_{m}^{b} \frac{\partial M_{n}}{\partial \lambda_{m}^{a}}\right) \frac{\partial}{\partial P^{b \dot{a}}} \delta^{(4)}(P)\right) .
\end{aligned}
$$

The last sum can be evaluated further:

$$
\sum_{m} \lambda_{m}^{b} \frac{\partial M_{n}}{\partial \lambda_{m}^{a}}=\frac{1}{2} \delta_{a}^{b} \sum_{m} \lambda_{m}^{c} \frac{\partial M_{n}}{\partial \lambda_{m}^{c}}
$$

where

$$
\sum_{m=1}^{n} \lambda_{m}^{c} \frac{\partial M_{n}}{\partial \lambda_{m}^{c}}=(-2(n-3)+2-2 s+2 s) M_{n}=-2(n-4) M_{n}
$$

and plugging these expressions back into (4.32) yields $K_{a \dot{a}} \hat{M}_{n}=0$. Similar calculation for the amplitude (3.28) gives

$$
\sum_{m=1}^{n} \lambda_{m}^{c} \frac{\partial M_{n}}{\partial \lambda_{m}^{c}}=\left(-2(n-4)+2 s_{1}-2 s_{1}+2 s_{2}-2 s_{2}\right) M_{n}=-2(n-4) M_{n} .
$$


Thus the generalized tree amplitudes (3.24) and (3.28) are conformally invariant. As it is easy to check the four graviton scattering amplitude (3.21) is not conformally invariant because of the spinor product $[1,3]$ in (3.21). This is another manifestation of the difference between charge and neutral helicity-two particles which has its origin in the structure of the corresponding vertices (2.16),$(2.17)$ and (2.18) .

We do not know whether all tree level amplitudes of high spin gauge theory are conformally invariant or not. The reason is that presently there are very limited higher spin amplitudes which are available for the analyzes. The on-shell scattering amplitudes (3.24) and (3.28) became available only thanks to the new recursion relations for the tree-level amplitudes for the lower spin gauge theories set up in [4, 15, 6, 17, 8, 19, 10, 11, 17, 18, 19, 20, 21, 22, 23, 24, 25]. The problem of conformal invariance of higher spin gauge theories is an old and unsolved problem even at the classical level and the above result can shed some light on that difficult problem. The higher spin extension of the conformal group have been found recently in [3], but it is not yet known if we have here its field theoretical realization. The progress in the construction of multi-particle and multi-loop scattering amplitudes in $\mathcal{N}=4 \mathrm{SYM}$ in spinor and twistor spaces [60, 61, 62, 14, 15, 63] raises a hope that future progress can be reached also in higher spin gauge theories. It is difficult to expect that the scattering amplitudes of the tensor gauge bosons will remain conformal invariant at the loop-level and most probably we shall have the breaking of conformal symmetry similar to the QCD case.

We have to notice also that whenever two momenta become collinear, conformal symmetry becomes anomalous [12, 13, 14, 15] and the naive action of infinitesimal conformal transformations on scattering amplitudes is not complete [14, 15, 16]. It needs to be supplemented by correction terms which cure the collinear anomaly at tree level. The corrections have the ability to change the number of legs of the scattering amplitudes, so that such generators act on the generating functional of all amplitudes [16]. In the next section we shall study collinear momenta behavior of the scattering amplitudes of tensor gauge bosons and shall compare it with the YM case. 


\section{$5 \quad$ Collinear Behavior and Splitting Functions}

The color-ordered amplitudes in QCD can only have poles in channels of cyclically adjacent momenta [8]. But, in fact, only in two-particle channels the corresponding MHV amplitudes develop the poles, due to the vanishing of $M_{n}^{\text {tree }}\left(1^{ \pm}, 2^{+}, \ldots, n^{+}\right)$amplitudes. Thus only collinear (two-particle) singularities of adjacent particles are permitted and the collinear behavior of the tree amplitudes has the following general form [8, 9, 10, 27, 28]:

$$
M_{n}^{\text {tree }}\left(\ldots, a^{\lambda_{a}}, b^{\lambda_{b}}, \ldots\right) \frac{a \| b}{\rightarrow} \sum_{\lambda= \pm 1} \operatorname{Split}_{-\lambda}^{\text {tree }}\left(a^{\lambda_{a}}, b^{\lambda_{b}}\right) \times M_{n-1}^{\text {tree }}\left(\ldots, P^{\lambda}, \ldots\right)
$$

where $\operatorname{Split}_{-\lambda}^{\text {tree }}\left(a^{\lambda_{a}}, b^{\lambda_{b}}\right)$ denotes the splitting amplitude, the intermediate state $P$ has momentum $k_{P}=k_{a}+k_{b}$ and helicity $\lambda$.

In the generalized Yang-Mills theory the tree amplitudes with only one negative helicity are not vanishing $M_{n}^{\text {tree }}(-,+, \ldots,+) \neq 0$, as we have seen at the end of the third section (3.29). Therefore multi-particle pole can appear in the corresponding limit, if tensor bosons have different spins: let us say, $s$ and $s-2$. Bellow we shall consider only the case of equal spins. An easy way to extract the splitting amplitudes in that case will be to consider five-point amplitude (3.25). Let us consider the amplitude (3.25) in the limit when the particles 4 and 5 become collinear: $k_{4} \| k_{5}$, that is, $k_{4}=x k_{P}, k_{5}=(1-x) k_{P}, k_{P}^{2} \rightarrow 0$ and $x$ describes the longitudinal momentum sharing. In this limit we have

$$
\lambda_{4}=\sqrt{x} \lambda_{P}, \quad \lambda_{5}=\sqrt{1-x} \lambda_{P}
$$

and can bring the amplitude in the following form:

$$
\begin{aligned}
M_{5}\left(1^{+}, 2^{-}, 3^{+}, 4^{+s}, 5^{-s}\right) & =\frac{<2 P>^{4}}{<1,2><2,3><3, P><P, 1>} \frac{(1-x)^{s+1 / 2}}{x^{s-1 / 2}} \frac{1}{<4,5>}= \\
& =A_{4}\left(1^{+}, 2^{-}, 3^{+}, P^{-}\right) \times \text {Split }_{+}\left(a^{+s}, b^{-s}\right)
\end{aligned}
$$

where

$$
\operatorname{Split}_{+}\left(a^{+s}, b^{-s}\right)=\left(\frac{1-x}{x}\right)^{s-1} \frac{(1-x)^{2}}{\sqrt{x(1-x)}} \frac{1}{<a, b>}
$$

and one can deduce that

$$
\operatorname{Split}_{+}\left(a^{-s}, b^{+s}\right)=\left(\frac{x}{1-x}\right)^{s-1} \frac{x^{2}}{\sqrt{x(1-x)}} \frac{1}{<a, b>} .
$$


Considering different collinear limits $k_{1} \| k_{5}$ and $k_{3} \| k_{4}$ one can get

$$
\begin{aligned}
& \operatorname{Split}_{+s}\left(a^{+}, b^{-s}\right)=(1-x)^{s-1} \frac{(1-x)^{2}}{\sqrt{x(1-x)}} \frac{1}{<a, b>}, \\
& \operatorname{Split}_{+s}\left(a^{-s}, b^{+}\right)=x^{s-1} \frac{x^{2}}{\sqrt{x(1-x)}} \frac{1}{<a, b>}
\end{aligned}
$$

and

$$
\begin{aligned}
& \operatorname{Split}_{-s}\left(a^{+s}, b^{+}\right)=\left(\frac{1}{x}\right)^{s-1} \frac{1}{\sqrt{x(1-x)}} \frac{1}{<a, b>}, \\
& \text { Split }_{-s}\left(a^{+}, b^{+s}\right)=\left(\frac{1}{1-x}\right)^{s-1} \frac{1}{\sqrt{x(1-x)}} \frac{1}{<a, b>} .
\end{aligned}
$$

This set of splitting functions for the amplitudes $V \rightarrow T T$ and $T \rightarrow V T$ reduces to the gluon splitting functions [8, 9, 10, 27, 28] if one takes $s=1$. The residue of the collinear pole in the square of the factorized amplitude gives the probability of creating a pair of tensor gauge bosons

$$
\sigma^{n g T T}=\sigma^{(n-1) g} \times P_{g T T}(x)
$$

where

$$
P_{g T T}(x)=\left(\frac{x^{2}}{\sqrt{x(1-x)}}\right)^{2}\left(\frac{x}{1-x}\right)^{2 s-2}+\left(\frac{(1-x)^{2}}{\sqrt{x(1-x)}}\right)^{2}\left(\frac{1-x}{x}\right)^{2 s-2},
$$

with strong amplification of the forward and backward creation probability. We shall consider the multi-particle pole structure in a future work.

\section{Acknowledgement}

We would like to thank L. Brink, L. J. Dixon, J. M. Drummond and T. R. Taylor for helpful discussions. One of us, G.S., would like to thank the CERN Theory Division for hospitality and G. Georgiou for discussions and participation in the early stages of this work. This work was supported in part by the European Commission under the ERC Advanced Grant 226371 and the contract PITN-GA-2009-237920.

\section{References}

[1] G. Savvidy, Non-Abelian tensor gauge fields: Generalization of Yang-Mills theory, Phys. Lett. B 625 (2005) 341 
[2] G. Savvidy, Extension of the Poincaré Group and Non-Abelian Tensor Gauge Fields, Int. J. Mod. Phys. A 25 (2010) 5765 [arXiv:1006.3005 [hep-th]].

[3] I. Antoniadis, L. Brink and G. Savvidy, Extensions of the Poincare group, J. Math. Phys. 52 (2011) 072303 arXiv:1103.2456 [hep-th]].

[4] F. A. Berends, R. Kleiss, P. De Causmaecker, R. Gastmans and T. T. Wu, Single Bremsstrahlung Processes In Gauge Theories, Phys. Lett. B 103 (1981) 124.

[5] R. Kleiss and W. J. Stirling, Spinor Techniques For Calculating $P$ Anti-P $\rightarrow W^{+-} / Z^{0}$ + Jets, Nucl. Phys. B 262 (1985) 235.

[6] Z. Xu, D. H. Zhang and L. Chang, Helicity Amplitudes for Multiple Bremsstrahlung in Massless Nonabelian Gauge Theories, Nucl. Phys. B 291 (1987) 392.

[7] J. F. Gunion and Z. Kunszt, Improved Analytic Techniques For Tree Graph Calculations And The GGQ Anti-Q Lepton Anti-Lepton Subprocess, Phys. Lett. B 161 (1985) 333.

[8] L. J. Dixon, Calculating scattering amplitudes efficiently, arXiv:hep-ph/9601359.

[9] S. J. Parke and T. R. Taylor, An Amplitude for $n$ Gluon Scattering, Phys. Rev. Lett. 56 (1986) 2459.

[10] F. A. Berends and W. T. Giele, Recursive Calculations for Processes with $n$ Gluons, Nucl. Phys. B 306 (1988) 759.

[11] E. Witten, Perturbative gauge theory as a string theory in twistor space, Commun. Math. Phys. 252 (2004) 189 [arXiv:hep-th/0312171].

[12] F. Cachazo, P. Svrcek and E. Witten, Gauge theory amplitudes in twistor space and holomorphic anomaly, JHEP 0410 (2004) 077 hep-th/0409245].

[13] F. Cachazo, Holomorphic anomaly of unitarity cuts and one-loop gauge theory amplitudes, hep-th/0410077.

[14] L. J. Mason and D. Skinner, Scattering Amplitudes and BCFW Recursion in Twistor Space, JHEP 1001 (2010) 064 [arXiv:0903.2083 [hep-th]]. 
[15] N. Arkani-Hamed, F. Cachazo, C. Cheung and J. Kaplan, The S-Matrix in Twistor Space, JHEP 1003 (2010) 110 [arXiv:0903.2110 [hep-th]].

[16] T. Bargheer, N. Beisert, W. Galleas, F. Loebbert and T. McLoughlin, Exacting N=4 Superconformal Symmetry, JHEP 0911 (2009) 056 [arXiv:0905.3738 [hep-th]].

[17] R. Britto, F. Cachazo and B. Feng, New recursion relations for tree amplitudes of gluons, Nucl. Phys. B 715 (2005) 499 arXiv:hep-th/0412308.

[18] R. Britto, F. Cachazo, B. Feng and E. Witten, Direct proof of tree-level recursion relation in Yang-Mills theory, Phys. Rev. Lett. 94 (2005) 181602 [arXiv:hep-th/0501052].

[19] P. Benincasa and F. Cachazo, Consistency Conditions on the S-Matrix of Massless Particles, arXiv:0705.4305 [hep-th].

[20] F. Cachazo, P. Svrcek and E. Witten, MHV vertices and tree amplitudes in gauge theory, JHEP 0409 (2004) 006 [arXiv:hep-th/0403047].

[21] G. Georgiou, E. W. N. Glover and V. V. Khoze, Non-MHV Tree Amplitudes in Gauge Theory, JHEP 0407 (2004) 048 [arXiv:hep-th/0407027].

[22] G. Georgiou and V. V. Khoze, Tree amplitudes in gauge theory as scalar MHV diagrams, JHEP 0405 (2004) 070 arXiv:hep-th/0404072.

[23] J. Bedford, A. Brandhuber, B. J. Spence and G. Travaglini, A recursion relation for gravity amplitudes, Nucl. Phys. B 721 (2005) 98 arXiv:hep-th/0502146.

[24] F. Cachazo and P. Svrcek, Tree level recursion relations in general relativity, arXiv:hep-th/0502160.

[25] N. Arkani-Hamed and J. Kaplan, On Tree Amplitudes in Gauge Theory and Gravity, JHEP 0804 (2008) 076 arXiv:0801.2385 [hep-th]].

[26] G. Georgiou and G. Savvidy, Production of non-Abelian tensor gauge bosons. Tree amplitudes and BCFW recursion relation, Int. J. Mod. Phys. A 26 (2011) 2537 arXiv:1007.3756 [hep-th]].

[27] F. A. Berends and W. T. Giele, Multiple Soft Gluon Radiation in Parton Processes, Nucl. Phys. B 313 (1989) 595. 
[28] M. L. Mangano and S. J. Parke, Quark - Gluon Amplitudes in the Dual Expansion, Nucl. Phys. B 299 (1988) 673.

[29] J. Polchinski, Scale And Conformal Invariance In Quantum Field Theory, Nucl. Phys. B 303 (1988) 226.

[30] A. B. Zamolodchikov, Irreversibility of the Flux of the Renormalization Group in a 2D Field Theory, JETP Lett. 43 (1986) 730 [Pisma Zh. Eksp. Teor. Fiz. 43 (1986) $565]$.

[31] J. L. Cardy, Is There a c Theorem in Four-Dimensions?, Phys. Lett. B 215 (1988) 749 .

[32] I. Antoniadis and M. Buican, On R-symmetric Fixed Points and Superconformality, Phys. Rev. D 83 (2011) 105011 [arXiv:1102.2294 [hep-th]].

[33] S. El-Showk, Y. Nakayama and S. Rychkov, What Maxwell Theory in $D<>4$ teaches us about scale and conformal invariance, Nucl. Phys. B 848 (2011) 578 arXiv:1101.5385 [hep-th]].

[34] J. F. Fortin, B. Grinstein and A. Stergiou, Scale without Conformal Invariance: An Example, arXiv:1106.2540 [hep-th].

[35] J. F. Fortin, B. Grinstein and A. Stergiou, Scale without Conformal Invariance: Theoretical Foundations, arXiv:1107.3840 [hep-th].

[36] A. K. Bengtsson, I. Bengtsson and L. Brink, Cubic Interaction Terms For Arbitrary Spin, Nucl. Phys. B 227 (1983) 31.

[37] A. K. Bengtsson, I. Bengtsson and L. Brink, Cubic Interaction Terms For Arbitrarily Extended Supermultiplets, Nucl. Phys. B 227 (1983) 41.

[38] A. K. H. Bengtsson, I. Bengtsson and N. Linden, Interacting Higher Spin Gauge Fields on the Light Front, Class. Quant. Grav. 4 (1987) 1333.

[39] R. R. Metsaev, Cubic interaction vertices of massive and massless higher spin fields, Nucl. Phys. B 759 (2006) 147 
[40] F. A. Berends, G. J. H. Burgers and H. van Dam, On The Theoretical Problems In Constructing Interactions Involving Higher Spin Massless Particles, Nucl. Phys. B 260 (1985) 295.

[41] F. A. Berends, G. J. H. Burgers and H. van Dam, Explicit Construction Of Conserved Currents For Massless Fields Of Arbitrary Spin, Nucl. Phys. B 271 (1986) 429.

[42] F. A. Berends, G. J. H. Burgers and H. Van Dam, On Spin Three Selfinteractions, Z. Phys. C 24 (1984) 247.

[43] J.Schwinger. Particles, Sourses, and Fields (Addison-Wesley, Reading, MA, 1970)

[44] C.Fronsdal. Massless fields with integer spin, Phys.Rev. D18 (1978) 3624

[45] S. Guttenberg and G. Savvidy, Schwinger-Fronsdal Theory of Abelian Tensor Gauge Fields, SIGMA 4 (2008) 061 [arXiv:0804.0522 [hep-th]].

[46] M. Fierz. Über die relativistische Theorie kräftefreier Teilchen mit beliebigem Spin. Helv. Phys. Acta. 12 (1939) 3.

[47] M. Fierz and W. Pauli. On Relativistic Wave Equations for Particles of Arbitrary Spin in an Electromagnetic Field. Proc. Roy. Soc. A173 (1939) 211.

[48] W. Rarita and J. Schwinger. On a Theory of Particles with Half-Integral Spin. Phys. Rev. 60 (1941) 61

[49] L. P. S. Singh and C. R. Hagen. Lagrangian formulation for arbitrary spin. I. The boson case. Phys. Rev. D9 (1974) 898

[50] L. P. S. Singh and C. R. Hagen. Lagrangian formulation for arbitrary spin. II. The fermion case. Phys. Rev. D9 (1974) 898, 910

[51] J.Fang and C.Fronsdal. Massless fields with half-integral spin, Phys. Rev. D18 (1978) 3630

[52] T. Curtright, Generalized Gauge Fields, Phys. Lett. B165 (1985) 304

[53] S.Deser. Self-interaction and gauge invariance. Gen. Rel. Grav. 1, (1970) 9. 
[54] H. Kawai, D. C. Lewellen and S. H. H. Tye, A Relation Between Tree Amplitudes Of Closed And Open Strings, Nucl. Phys. B 269 (1986) 1.

[55] F. A. Berends, W. T. Giele and H. Kuijf, On relations between multi - gluon and multigraviton scattering, Phys. Lett. B 211 (1988) 91.

[56] G. Savvidy, Non-abelian tensor gauge fields. I, Int. J. Mod. Phys. A 21 (2006) 4931;

[57] G. Savvidy, Non-abelian tensor gauge fields. II, Int. J. Mod. Phys. A 21 (2006) 4959;

[58] M. T. Grisaru, H. N. Pendleton, P. van Nieuwenhuizen, Supergravity and the S Matrix, Phys. Rev. D15 (1977) 996.

[59] M. T. Grisaru, H. N. Pendleton, Some Properties of Scattering Amplitudes in Supersymmetric Theories, Nucl. Phys. B124, 81 (1977).

[60] V. P. Nair, A Current Algebra For Some Gauge Theory Amplitudes, Phys. Lett. B 214 (1988) 215.

[61] J. M. Drummond and J. M. Henn, All tree-level amplitudes in N=4 SYM, JHEP 0904, 018 (2009) [arXiv:0808.2475 [hep-th]].

[62] J. M. Drummond, J. M. Henn and J. Plefka, Yangian symmetry of scattering amplitudes in N=4 super Yang-Mills theory, JHEP 0905 (2009) 046 arXiv:0902.2987 [hep-th]].

[63] J. M. Drummond, J. Henn, G. P. Korchemsky and E. Sokatchev, Dual superconformal symmetry of scattering amplitudes in N=4 super-Yang-Mills theory, Nucl. Phys. B 828 (2010) 317 [arXiv:0807.1095 [hep-th]]. 